\title{
Aplicación móvil en realidad aumentada para la formación y comunicación de niños con Síndrome de
}

Down

Mobile application in increased reality for the training and communication of children with down syndrome

Hernán Darío Centeno Aulla. ${ }^{1}$, Lourdes Emperatriz Paredes Castelo. ${ }^{2}$ Marcelo Eduardo Allauca Peñafiel. ${ }^{3}$, Luis Gonzalo Allauca Peñafiel. ${ }^{4}$ \& Lady Marieliza Espinoza

Tinoco. ${ }^{5}$

\section{Recibido: 21-05-2020 /Aceptado: 20-06-2020 / Publicado: 03-07-2020}

\begin{abstract}
DOI: $\underline{\text { https://doi.org/10.33262/concienciadigital.v3i3.1307 }}$
Abstract.

The objective of this research was to apply a mobile system in Augmented Reality to support the training and communication of children with Down syndrome. For this, a learning support tool is found with the assistance of professionals specialized in the area, through an application in Augmented Reality with 3D environments in pictograms. The application was aligned in parameters established in the ISO / IEC 9126 standard; among them: functionality, reliability, usability, efficiency, maintainability and portability. The research is descriptive, field and bibliographic. The population and sample were intentional, consisting of 33 children with Down syndrome. To obtain the data, the survey technique is needed, using the Likert scale in order to quantify the degree of conformity of the parents on the interactivity of their children with the application of Augmented Reality. The data obtained were tabulated, analyzed and interpreted and allowed to know

\footnotetext{
${ }^{1}$ Escuela Superior Politécnica de Chimborazo, Departamento de Tecnología de Información y Comunicación, Riobamba, Ecuador, hernan.centeno@espoch.edu.ec

${ }^{2}$ Escuela Superior Politécnica de Chimborazo, Facultad de Ciencias, Riobamba, Ecuador, lparedes@espoch.edu.ec

${ }^{3}$ Escuela Superior Politécnica de Chimborazo, Centro de Idiomas, Riobamba, Ecuador, mallauca@espoch.edu.ec

${ }^{4}$ Universidad Nacional de Chimborazo, Facultad de Ingeniería, Riobamba, Ecuador, gallauca@unach.edu.ec

${ }^{5}$ Universidad Nacional de Chimborazo, Facultad de Ingeniería, Riobamba, Ecuador, lespinoza@unach.edu.ec
} 
the use of Augmented Reality dramatically attracts their attention and positive changes in the interaction of children with Down Syndrome. In other words, the technological tools identified in the support in their communication and training. The objective of this research was to apply a mobile system in Augmented Reality to support the training and communication of children with Down syndrome. For this, a learning support tool is found with the assistance of professionals specialized in the area, through an application in Augmented Reality with 3D environments in pictograms. The application was aligned in parameters established in the ISO / IEC 9126 standard; among them: functionality, reliability, usability, efficiency, maintainability and portability. The research is descriptive, field and bibliographic. The population and sample were intentional, consisting of 33 children with Down syndrome. To obtain the data, the survey technique is needed, using the Likert scale in order to quantify the degree of conformity of the parents on the interactivity of their children with the application of Augmented Reality. The data obtained were tabulated, analyzed and interpreted and allowed to know the use of Augmented Reality dramatically attracts their attention and positive changes in the interaction of children with Down Syndrome. In other words, the technological tools identified in the support in their communication and training.

Keywords: Mobile application, Augmented Reality, Down Syndrome, Education, Communication System.

\section{Resumen.}

El objetivo de la presente investigación fue aplicar un sistema móvil en Realidad Aumentada para apoyar en la formación y comunicación de niños con síndrome de Down. Para ello, se desarrolló con la asistencia de profesionales especialistas en el área una herramienta de apoyo de aprendizaje, mediante una aplicación en Realidad Aumentada con entornos 3D basados en pictogramas. La aplicación se alineó en parámetros establecidos en el estándar ISO/IEC 9126; entre ellos: funcionalidad, fiabilidad, usabilidad, eficiencia, mantenibilidad y portabilidad. La investigación es descriptiva, de campo y bibliográfica. La población y muestra fueron intencionales, constituida por 33 niños con síndrome de Down. Para la obtención de los datos se utilizó la técnica de la encuesta, utilizando la escala de Likert a fin en cuantificar el grado de conformidad de los padres de familia sobre la interactividad de sus niños con la aplicación de Realidad Aumentada. Los datos obtenidos fueron tabulados, analizados e interpretaron y permitieron conocer que el uso de la Realidad Aumentada atrae notablemente su atención y reflejan cambios positivos en la interacción de los niños con Síndrome de Down. Es decir que las herramientas tecnológicas contribuyen en el apoyo en su comunicación y formación.

Palabras clave: Aplicación móvil, Realidad aumentada, Síndrome Down, Educación, Sistema de comunicación. 


\section{Introducción.}

Los niños con síndrome Down, presentan problemas en la expresión del lenguaje (acto de hablar) o producción del lenguaje; es decir dificultades en las primeras etapas, para la adquisición y producción de fonemas que son características específicas que impiden un lenguaje fácil de entender e ininteligible. (reeduca.com, 2009)

Por otra parte, desde el punto de vista educativo Ortega \& Gómez-Ariza (2005) sostienen que un objetivo deseable en el aprendizaje de los niños Down, es permitir la generalización de conocimientos durante el proceso de adquisición en contextos distintos de los normalmente utilizados; pues los niños Down (SD) presentan serias dificultades de generalización.

En la actualidad existen sistemas de comunicación alternativos aumentativos, entre ellos el uso de pictogramas con imágenes inanimadas. En instituciones de educación especial suelen utilizar regularmente el Sistema 2D (SiDow), ya que contienen una gama amplia de pictogramas en blanco y negro con entornos llamativos, pero carece del tridimensionalismo que si soporta o implementa la Realidad Aumentada (3D).

Los niños, ya sean normales o con síndrome de Down, tienen interés en aprender y explorar cosas nuevas; y sin duda las nuevas tecnologías de la información y comunicación permiten retención de conocimiento y al mismo tiempo son instrumentos motivadores a través de las representaciones imágenes agradables con interfaces intuitiva que permiten intuir los pasos a seguir para llegar a un objetivo.

En la investigación se propone usar una estrategia de aprendizaje gracias a la implementación de un sistema móvil con Realidad Aumentada, soportados en un motor de desarrollo Unity de Unity Technologies, Blender (libre) y Vuforia de Qualcom.

Según Ouazzani (2012) el motor y potencia de Unity permite obtener resultados de máxima calidad, con una inversión mínimo de esfuerzo, gracias a sus nuevas funciones, actualizaciones y mejoras de inclusión permiten un procesamiento y desarrollo óptimo para la obtención de los productos finales de animación sigue en curso. Unity ofrece su versión profesional adquiriendo un pago previo y también pone a disposición una versión libre que es gratuita y que se puede descargar desde su página Web, pero al ser gratuita tiene menos funcionalidades, sin embrago permite la creación de videojuegos de muy buena calidad.

Vuforia, es una aplicación de desarrollo de software que permite la implementación de aplicaciones móviles, que contiene un motor potente de reconocimiento de imágenes, así como un amplio conjunto de herramientas que permite apoyar la creatividad en el área de objetos de diseo gráfico. Además, es una aplicación multiplataforma, es decir es compatible con Android, iOS, es decir que permite la adaptación de la aplicación con diversos dispositivos si presentar dificultad de visualización y uso (Qualcomm, 2010) 
En cuanto a Blender, se le considera como una aplicación orientado al modelado 3D de objetos, para posterior realizar representaciones de los mismos modelados. Es decir que se puede dar características a los objetos 3D tales como: texturas y materiales, iluminación de la escena, etc. En su frame de trabajo incluye tecnologías que son muy frecuente y utilizadas en diseño 3D; tales como: textos, mallas, curvas, meta-objetos, modelado escultórico y superficies. Mediante el el uso de pictogramas en blanco y negro puede aplicarse marcadores que se describen en un objeto animado 3D.

La metodología utilizada fue XP, ya que es una metodología clave para el éxito en desarrollo de software; pues es centrada y ágil, la misma que permite potenciar las relaciones interpersonales, promueve el trabajo colaborativo o en equipos y tiene como aporte el aprendizaje de los desarrolladores. La metodología propicia un buen clima de trabajo y permite feedback continuo entre el equipo de desarrollo y el cliente, es decir desarrolla una comunicación fluida entre los participantes, simplificación de soluciones y alternativas para enfrentar los cambios. (Letelier \& Penadés, 2006)

Vuforia permitirá plantear una propuesta donde se usarán marcados RA a través de los pictogramas; y con el diseño de un personaje en 3D realizado mediante blender, se emitirá mensajes cuando se enfoque la cámara Smartphone a un determinado pictograma. El personaje en 3D se diseñó en blender y para animarlo y exportarlo se utilizará Unity.

Según NICHCY (2010) sostiene que el síndrome de Down es la condición asociada con el retraso mental de los niños y es común y fácil de reconocer. Dicha condición que antes fue conocida como mongolismo, fue el resultado de una anormalidad de los cromosomas: de razón inexplicable que representa una desviación en la producción de 47 cromosomas en lugar de 46 que se consideran normales en una célula. Por lo que se ha identificado que el cromosoma adicional, afecta en el cambia total del desarrollo ordenado del cerebro y del cuerpo. La identificación de este síndrome, se obtiene por el diagnóstico mediante una prueba de cromosomas realizada poco después del nacimiento del niño.

Por otra parte, Albuja (2015) sostiene que el niño con síndrome de Down, no puede sostener la atención por largo tiempo y se fatiga rápidamente; por tanto, para interactuar con los niños Down, se debe trabajar con períodos cortos que deben ir prolongándose de forma progresiva y de poco a poco. Las habilidades que presentan serias carencias son la imaginación y flexibilidad, por lo que los educadores deben aportar significativamente en sus procesos (Rodríguez, 2011)

Marcelo Varela secretario general de la Asociación Síndrome de Down de Argentina (ASDRA), sostiene que "No podemos pretender que, en una era digital, los chicos con discapacidad sigan aprendiendo con papel y lápiz".

Cavanna (2016) indica que el aprendizaje digital, que se basa en apoyos visuales y sonoros, incrementa la capacidad de almacenamiento y procesamiento de información de los alumnos, posibilita una mejor comprensión de lo abstracto, fomenta la reflexión, y 
permite corregir trastornos del lenguaje. Además, esta metodología refuerza la visión, la audición y la coordinación motriz del niño, entre otras ventajas.

Los niños Down aprenden palabras relacionadas con personas cercanas. Por eso se trabaja con una especie de cartas (pictogramas) en donde están las fotos de su familia y también los nombres en la parte de abajo. Luego, hay varios ejercicios. Las palabras que se le proponen para leer son las que el niño conoce en su vida real.

Almazán (2009) sostiene que los sistemas alternativos o aumentativos de comunicación contienen mecanismos de expresión y comprensión que son diferentes a la palabra hablada que contienen técnicas o recursos que pueden ser naturales o artificiales; mientras que Álvarez (2013) indica que un Sistema Alternativo y/o Aumentativo representan una variedad convencional de signos arbitrarios caracterizadas por un conjunto de reglas que permiten combinar los símbolos a fin de representar ideas que representan el mundo con propósito comunicativo. Por otra parte, Almazán (2009) sostiene que un Sistemas Pictográficos de Comunicación (SPC) contiene pictogramas que están representado por dibujos blancos sobre un fondo negro y una palabra escrita en blanco, es decir combinan símbolos pictográficos e ideográficos.

Figura 1: Sistema Pictográfico

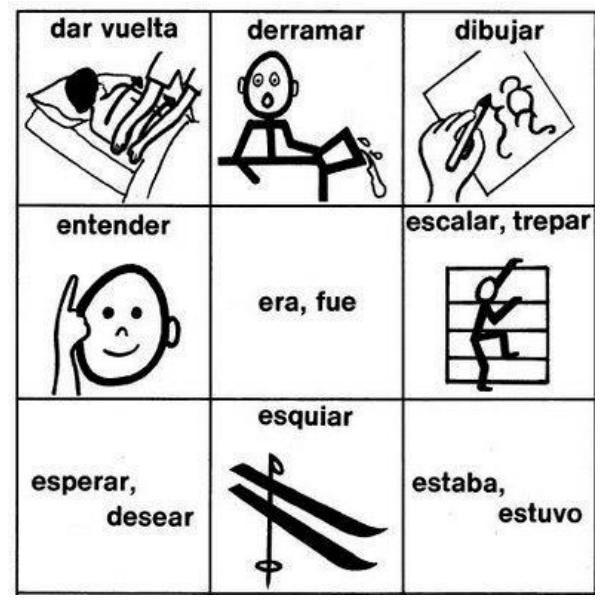

Fuente: (leyvrevh, 2012)

Según Basil (2015) los SPC pueden ser aplicados a personas analfabetas (por edad o discapacidad), con la característica que pueden aplicarlos a un nivel muy básico de comunicación, es decir, son adaptables y aplicados en niveles cognitivos bajos, en etapas iniciales o en un nivel de comunicación muy rico y avanzado, Con el atenuante que nunca pueden complementar y tener características de flexibilidad con respecto al que se puede alcanzar con el uso de la lengua escrita.

Por otra parte, Góngora (2011) sostiene que un pictograma puede aplicarse una persona cuando se dispone de habilidades mínimas tales como: buena agudeza visual y perceptiva, mínimo de intención comunicativa y mínimas habilidades cognitivas. 
Innovae (2016) sostiene que la realidad aumentada, permite combinar el mundo real con el virtual, mediante una interfaz, es decir, mediante un proceso informático que permite mejora la calidad de comunicación y enriquecer la experiencia visual.

Figura 2: Realidad Aumentada

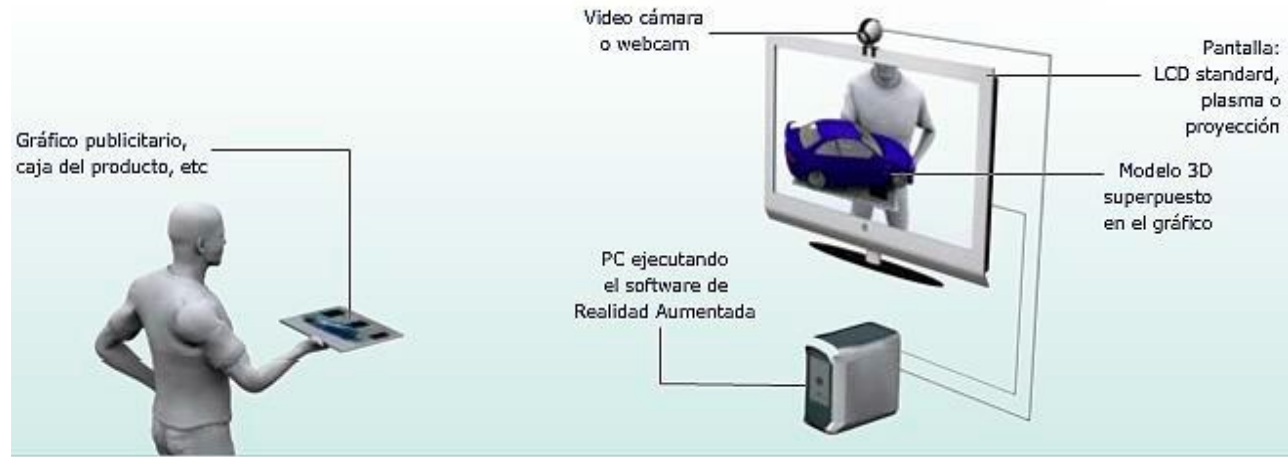

Fuente: (innovae, 2016)

Los niños con Síndrome de Down para su formación y comunicación pueden utilizar libros con pictogramas en blanco y negro, con imágenes inanimadas y nada llamativas, que representan recurso que pocos llamativos y nada motivadores. Por ello, se propone utilizar pictogramas con marcadores que permitan el uso de realidad aumentada, para que el niño con Síndrome de Down mediante el uso de un Smartphone y el enfoque del pictograma pueda observar un objeto en $3 \mathrm{D}$, que contenga video y animación que emita un mensaje. La propuesta de este sistema permitirá una mejor visión, a fin que el niño se involucre con el tópico y pueda retener con mayor facilidad la información.

Las innovaciones tecnologías han permitido potenciar el aprendizaje y la inclusión social de las niños y personas con síndrome de Down, y son ideales por, sobre todo, cuando tienen algunas características psicológicas y de aprendizaje propias de las personas con discapacidad intelectual. Por otra parte, estos niños son interactivos, exploradores y se auto motivan para conocer cosas nuevas y objetos; por ejemplo: un teléfono móvil, su manejo que puede resultar para ellos algo fácil y sencillo, ya que en un teléfono encuentran imágenes llamativas, animaciones, variedad de colores, videos, app que desembocan en motivar su interés y atracción, descartando limitantes tecnológicos y tampoco es una barrera que vayan surgiendo nuevas app, pues se adaptan fácilmente y responden manipulándoles, explorándoles y adaptándose a sus funcionalidades.

La realidad aumentada es una tecnología que ha incursionado en la educación, por ser una herramienta útil y valiosa como herramienta interactiva amigable con un ambiente amigable y entornos 3D, para el uso entre el estudiante y el profesor. Su implementación incluye entornos 3D, imágenes llamativas e intuitivas que acaparan la atención del estudiante en diferentes escenarios como el aula de clase o su entorno para la facilidad la información adquirida. 
Al obtener la atención total del estudiante mediante entornos 3D y materiales didácticos llamativos y amigables incidirá en la educación de tal forma que facilitará la captación del conocimiento que está transmitiendo el docente, pues al contrario de ser una actividad aburrida se convierte en una manera divertida de aprendizaje para el estudiante.

Los niños con Síndrome de Down tienen la característica de explorar y la consta de aprender cosas nuevas; entre ellas el uso de nuevas tecnologías; entre ellas las móviles, pues pueden utilizarlas al igual que un niño normal, pues también retienen el conocimiento al visualizar una imagen agradable y pueden intuir los pasos que deben seguir con aplicación que llama su atención y despiertan el interés para usarlas. Por lo que, si encuentran una aplicación móvil agradable, sentirán el deseo de usarlas, ya que los niños con Síndrome de Down les gusta manipular cualquier tipo de teléfonos debido a que las aplicaciones contienen imágenes coloridas animadas y juegos llamativos.

Por tanto, el objetivo de la presente investigación fue desarrollar una aplicación móvil con realidad aumentada, compatible con dispositivos con sistemas operativos en Android que incorporaron un personaje o avatar en 3D, la aplicación estuvo basada en ña metodología aumentativa alternativa para niños con Síndrome de Down con el fin de que sirva como una herramienta de apoyo en su comunicación y formación.

\section{Metodología:}

La investigación es bibliográfica, descriptiva, temporal, de campo y con enfoque educacional, ya que la ejecución y obtención de los datos fueron obtenidos por los propios sujetos de la experimentación.

La planificación para la implementación de la aplicación, se basó en el modelo de desarrollo de software SCRUM por ser una metodología ágil y flexible para gestionar el desarrollo de software. Para el desarrollo del Sistema móvil en Realidad Aumentada, se establecieron cinco sprint haciendo referencia cada uno de ellos a los módulos contemplados en el sistema en la Tabla 1, donde se detalla cada sprint.

Tabla 1. Planificación de Sprint del proyecto.

\begin{tabular}{ll}
\hline Sprint & \multicolumn{1}{c}{ Descripción } \\
\hline Sprint 1 & Análisis y recolección de información. \\
Sprint 2 & Creación de marcadores de RA. \\
Sprint 3 & $\begin{array}{l}\text { Creación del avatar 3D y obtención de modelados } \\
\text { 3D. }\end{array}$ \\
Sprint 4 & Creación de los módulos de la aplicación en RA.
\end{tabular}

Fuente: Elaboración propia. 
Inicialmente se realizó un previo análisis de las necesidades con los docentes y niños con Síndrome de Down de las instituciones de educación especial, con el objetivo de establecer los requerimientos del sistema para posteriormente definir la arquitectura a usar, metodología de desarrollo de software, herramientas de software para programación y diseño de interfaces y dispositivos necesarios para las respectivas pruebas del aplicativo móvil en Realidad Aumentada.

La integración de marcadores de Realidad Aumentada que en este caso serán los mismos pictogramas usados en el método tradicional de aprendizaje usado en el instituto.

La implementación de la aplicación "SiDow1.1" (Aplicación en R.A.) la cual será usada en las terapias de lenguajes de los niños con Síndrome de Down ayudando en su comunicación y formación.

El diagrama de casos de uso del sistema móvil en RA se muestran las actividades que realizan el docente y el niño Down en sus actividades para su comunicación y formación haciendo uso del sistema móvil, en la que se destacan actividades como: el uso de pictogramas para el aprendizaje del niño Down, el uso de tableros de comunicación, y el uso de la aplicativo realizado dando una mejor visión en entornos virtuales 3D en la figura 1 se puede visualizar el caso de uso y actores del sistema.

Figura 3. Diagrama de caso de uso y actores.

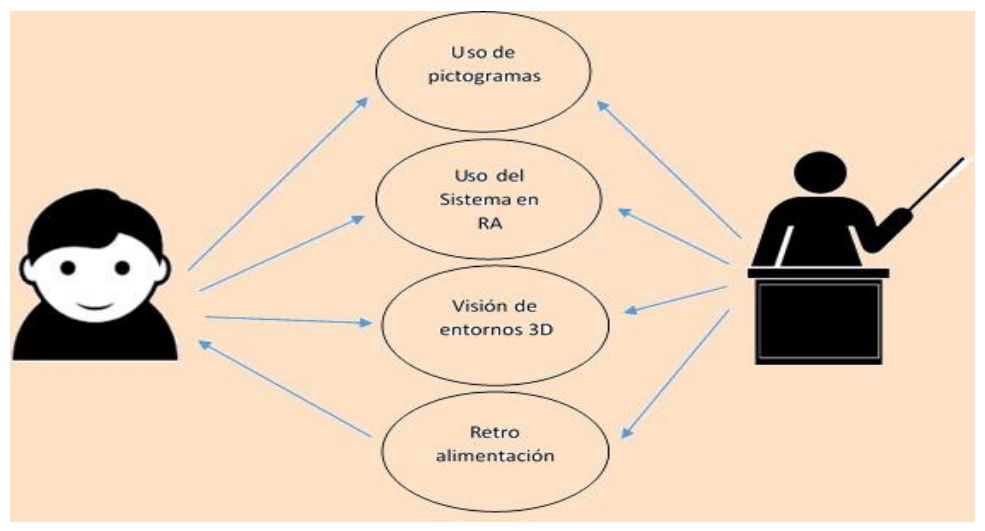

Fuente: Elaboración propia.

Los diagramas de casos de usos permiten modelar los requisitos de un sistema de software de manera tal que queden bien definidas las interacciones entre los usuarios y el sistema, y las interacciones entre el sistema en desarrollo y otros sistemas. Estos casos de uso son modelados empleando diagramas UML, los cuales poseen cierto conocimiento semántico u ontológico dentro de su estructura (Morales).

Se logró realizar la aplicación móvil con entornos en 3D y en Realidad Aumentada para niños con Síndrome de Down de las instituciones de educación especial el cual sirve de apoyo en la comunicación y formación de los mismos, interactuando con entornos 
virtuales en 3D haciendo un sistema de comunicación llamativo e intuitivo de usar, de esta manera se logra tener la atención del niño

La aplicación móvil en Realidad Aumentada está compuesta por 18. Cada módulo hace referencia a un determinado grupo de pictogramas como lo categorizan los docentes de educación especial de tal manera que al ingresar una escena especifica la aplicación solo mostrará los objetos 3D que hacen referencia a esa escena. Los módulos fueron clasificados de la siguiente manera: Pronombres, Sociales, Adjetivos, Verbos, Sustantivos, Alimentos, Bebidas, Frutas, Golosinas, Granos, Ropa, Juguetes, Tiempo, Diaz, Módulo de Meses, Colores, Números y Letras.

Figura 4. Estructura de Escenas.



Fuente: Elaboración propia.

Para el desarrollo de la aplicación móvil "SiDow1.1" se trabajando en Unity 3D haciendo uso de lenguaje javascript, además se incorporó el paquete de Vuforia para el reconocimiento de patrones creando así el aplicativo móvil en él se añadió los entornos 3D, cuenta con 18 módulos los mismos que corresponden a la clasificación de los pictogramas, en la figura 10-2 se puede apreciar la pantalla de inicio de la aplicación.

Figura 5. Pantalla de inicio de la aplicación.

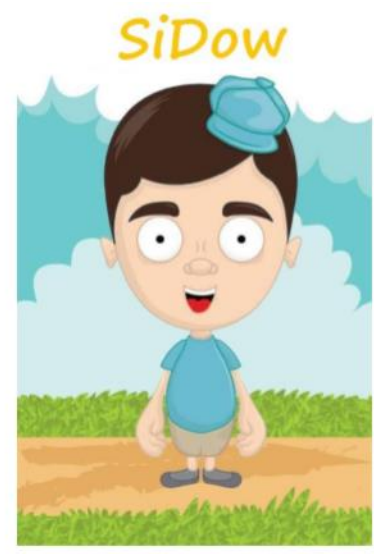

Fuente: Elaboración propia. 


\section{Población y muestra}

En la unidad educativa en donde se desarrolló la aplicación en realidad aumentada desarrollada cuenta con un total de 80 estudiantes, en este caso vendría a ser nuestro universo de estudio, con este valor se procede a calcular la muestra utilizando la fórmula estadística de una población conocida, suponiendo que los datos de la población se distribuyen normalmente.

$$
n=\frac{N * Z^{2} * p * q}{d^{2} *(N-1)+Z^{2} * p * q}
$$

En donde:

$N=$ Total de la Población.

$Z=1.96$ al cuadrado (si la seguridad es del 95\%)

$p=$ proporción esperada (en esta caso 5\% = 0.05)

$q=(1-p)$

$d=$ presición

Ahora esta fórmula la reemplazamos con los datos para poder determinar la muestra que se utilizará.

$$
\begin{gathered}
n=\frac{80 *(1.96)^{2} * 0.5 * 0.5}{0.05^{2} *(80-1)+1.96^{2} * 0.5 * 0.5} \\
\mathrm{n}=66.35
\end{gathered}
$$

Si $n N>10 \%$ la muestra debe ser corregida.

$66 / 80>10 \% ; \quad 0.825>100 \% ; \quad 83 \%>100 \%$;

En este caso el valor de la muestra es de 66 personas que equivale a más del $10 \%$ por ciento de la población total por lo tanto la muestra debe ser corregida con otra fórmula.

$$
\begin{gathered}
n c=\frac{N \cdot n}{N+(n-1)} \\
n c=\frac{80 * 66}{80+(66-1)} ; \quad n c=33.41
\end{gathered}
$$

Después de haber aplicado la fórmula mencionada el valor de la muestra es de 33.41, redondeando el valor de la muestra queda 33 lo que equivale al número de niños que serán evaluados al hacer uso de la aplicación.

El desarrolló de la aplicación móvil con realidad aumentada, se basó en pictogramas que fueron utilizados en las clases como herramientas de apoyo en el proceso de formación y 
comunicación de los niños con síndrome de Down. La aplicación móvil incluye un avatar en $3 \mathrm{D}$ que se activa cuando reconoce un patrón y simula a través de movimiento la representación del pictograma, induciendo al niño a interpretar con mayor facilidad el significado.

El instrumento de recolección de datos, fue una encuesta de 8 preguntas dirigidas a los padres de familia de los niños con síndrome de Down. El instrumento fue elaborado y aprobado por especialistas del área en el contexto de la investigación, con un rango de opciones o criterio de evaluación basadas en una escala de Likert.

Una vez desarrolladas las actividades según un cronograma previsto en un plan de intervención, se procedió a aplicar la encuesta y posterior se filtraron, tabularon y representaron en tablas y gráficos, para proceder con el análisis e interpretación de resultados a fin de establecer las respectivas conclusiones del experimento de la investigación.

\section{Resultados.}

A continuación, el análisis e interpretación de la encuesta a los padres de familia de los niños del síndrome de Down, que se basaron en las siguientes preguntas según criterio de evaluación.

Tabla 2. Criterios de evaluación de la aplicación realizada

\begin{tabular}{|c|c|c|c|c|c|}
\hline \multirow[b]{2}{*}{ Preguntas } & \multicolumn{5}{|c|}{ Criterios de evaluación } \\
\hline & Bajo & $\begin{array}{c}\text { Medio } \\
\text { Bajo }\end{array}$ & Medio & $\begin{array}{l}\text { Medio } \\
\text { Alto }\end{array}$ & Alto \\
\hline $\begin{array}{l}\text { ¿Cómo considera el desempeño del niño con la } \\
\text { aplicación? }\end{array}$ & 0 & 3 & 7 & 10 & 13 \\
\hline $\begin{array}{l}\text { ¿Cómo considera la interacción del niño con los } \\
\text { gráficos de cada una de las aplicaciones? }\end{array}$ & 0 & 5 & 8 & 3 & 17 \\
\hline $\begin{array}{l}\text { ¿Cómo considera la reacción emocional del niño con } \\
\text { la aplicación? }\end{array}$ & 0 & 4 & 7 & 18 & 4 \\
\hline $\begin{array}{l}\text { ¿Cómo considera la interacción del niño con las } \\
\text { actividades cotidianas asociando con la aplicación? }\end{array}$ & 0 & 2 & 6 & 10 & 15 \\
\hline $\begin{array}{l}\text { ¿Cómo considera el desarrollo del aprendizaje del } \\
\text { niño con la aplicación? }\end{array}$ & 0 & 3 & 12 & 10 & 18 \\
\hline $\begin{array}{l}\text { ¿Cómo considera la facilidad de acceso a la } \\
\text { aplicación? }\end{array}$ & 0 & 5 & 6 & 12 & 10 \\
\hline $\begin{array}{l}\text { ¿Cómo considera el cambio de aprendizaje del niño } \\
\text { con la Realidad Aumentada? }\end{array}$ & 0 & 9 & 1 & 9 & 14 \\
\hline TOTAL & 0 & 31 & 47 & 72 & 91 \\
\hline PORCENTAJE & $0 \%$ & $12.86 \%$ & $19.50 \%$ & $29.88 \%$ & $37.75 \%$ \\
\hline
\end{tabular}

Fuente: Elaboración propia. 
Pregunta 1. ¿Cómo considera el desempeño del niño con la aplicación?

Tabla 3. Desempeño del niño con la aplicación

\begin{tabular}{lccccc}
\hline \multirow{2}{*}{ Preguntas } & Bajo & \multicolumn{3}{c}{ Criterios de evaluación } \\
& Medio & Medio & Medio & Alto \\
Bajo & & Alto & \\
\hline $\begin{array}{l}\text { ¿Cómo considera el desempeño del niño con la } \\
\text { aplicación? }\end{array}$ & 0 & 3 & 7 & 10 & 13 \\
\hline
\end{tabular}

Fuente: Elaboración propia.

Figura 6. Desempeño del niño con la aplicación

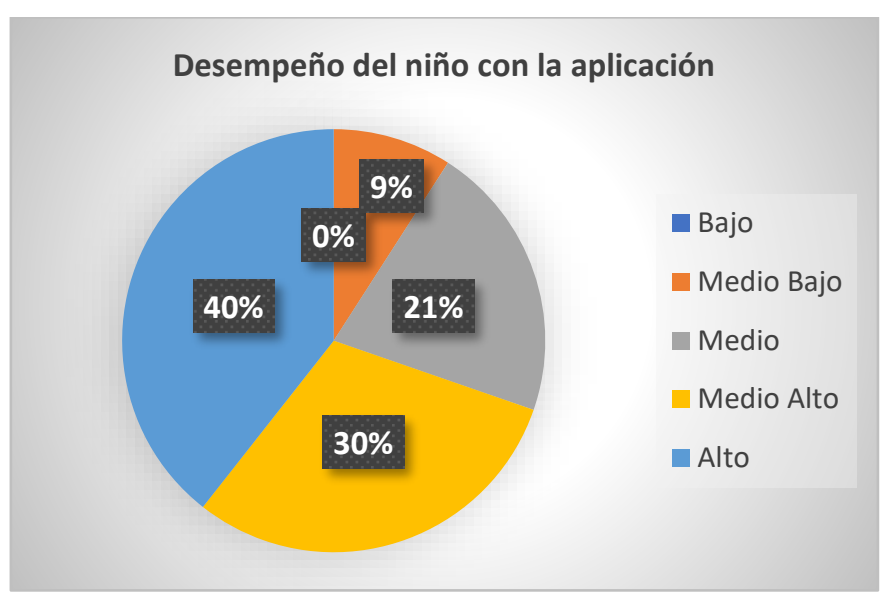

Fuente: Elaboración propia.

\section{Análisis e interpretación}

Del $100 \%$ de los padres de familia encuestados el $40 \%$ considera que el desempeño del niño con la aplicación es Alto, el 30\% Medio Alto, el 21\% Medio, el 9\% Medio Bajo; mientras que no se registraron frecuencias en la opción Bajo. Por lo que, la mayoría (opciones de Alto y Medio Alto) de padres de familia perciben que sus niños están experimentando nuevas formas de aprendizaje.

Pregunta 2. ¿Cómo considera la interacción del niño con los gráficos de cada una de las aplicaciones?

Tabla 4. Interacción del niño con los gráficos de cada una de las aplicaciones

\begin{tabular}{lccccc}
\hline \multicolumn{1}{c}{ Preguntas } & \multicolumn{5}{c}{ Criterios de evaluación } \\
& Bajo & $\begin{array}{c}\text { Medio } \\
\text { Bajo }\end{array}$ & Medio & $\begin{array}{c}\text { Medio } \\
\text { Alto }\end{array}$ & Alto \\
\hline $\begin{array}{l}\text { ¿Cómo considera la interacción del niño con los } \\
\text { gráficos de cada una de las aplicaciones? }\end{array}$ & 0 & 5 & 8 & 3 & 17 \\
\hline
\end{tabular}

Fuente: Elaboración propia. 
Figura 7. Interacción del niño con los gráficos de cada una de las aplicaciones

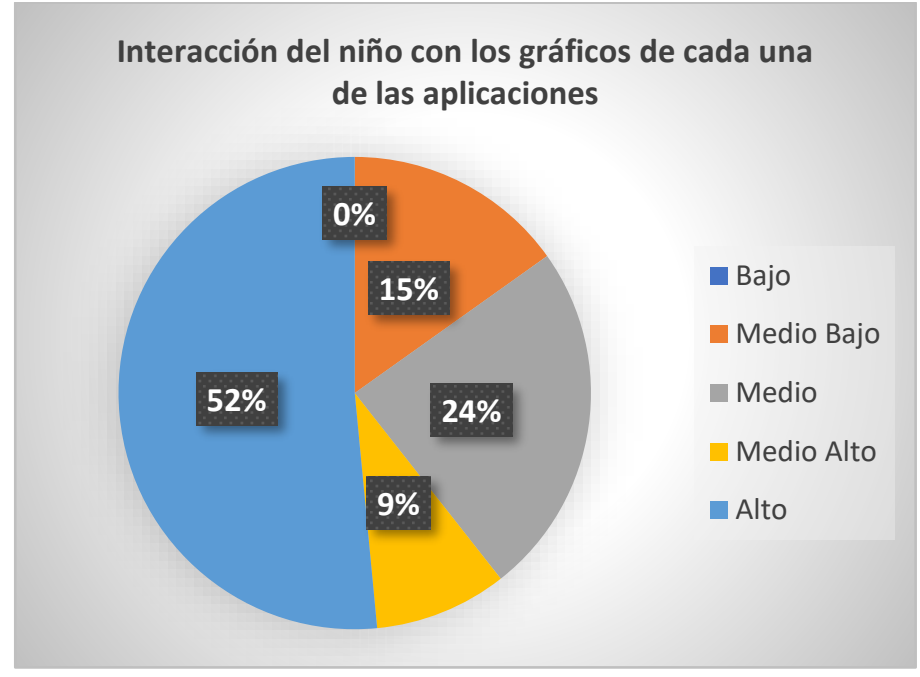

Fuente: Elaboración propia.

\section{Análisis e interpretación}

Del $100 \%$ de los padres de familia encuestados el 52\% considera que la interacción del niño con los gráficos de cada una de las aplicaciones es Alto, el 24\% Medio Alto, el 24\% Medio, el 9\% Medio Bajo mientras que no se registraron frecuencias en la opción Bajo. Es decir, que la mayoría (opciones de Alto y Medio Alto) de padres de familia consideran que sus niños interactuando de forma más dinámica que con los pictogramas tradicionales; ya que la tecnología (Realidad aumentada - 3D) induce a escenarios que llaman mejor su atención.

Pregunta 3. ¿Cómo considera la reacción emocional del niño con la aplicación?

Tabla 5. Reacción emocional del niño con la aplicación

\begin{tabular}{|c|c|c|c|c|c|}
\hline \multirow[b]{2}{*}{ Preguntas } & \multicolumn{5}{|c|}{ Criterios de evaluación } \\
\hline & Bajo & $\begin{array}{l}\text { Medio } \\
\text { Bajo }\end{array}$ & Medio & $\begin{array}{c}\text { Medio } \\
\text { Alto }\end{array}$ & Alto \\
\hline $\begin{array}{c}\text { ¿Cómo considera la reacción emocional del niño con la } \\
\text { aplicación? }\end{array}$ & 0 & 4 & 7 & 18 & 4 \\
\hline
\end{tabular}

Fuente: Elaboración propia. 
Figura 8. Reacción emocional del niño con la aplicación.

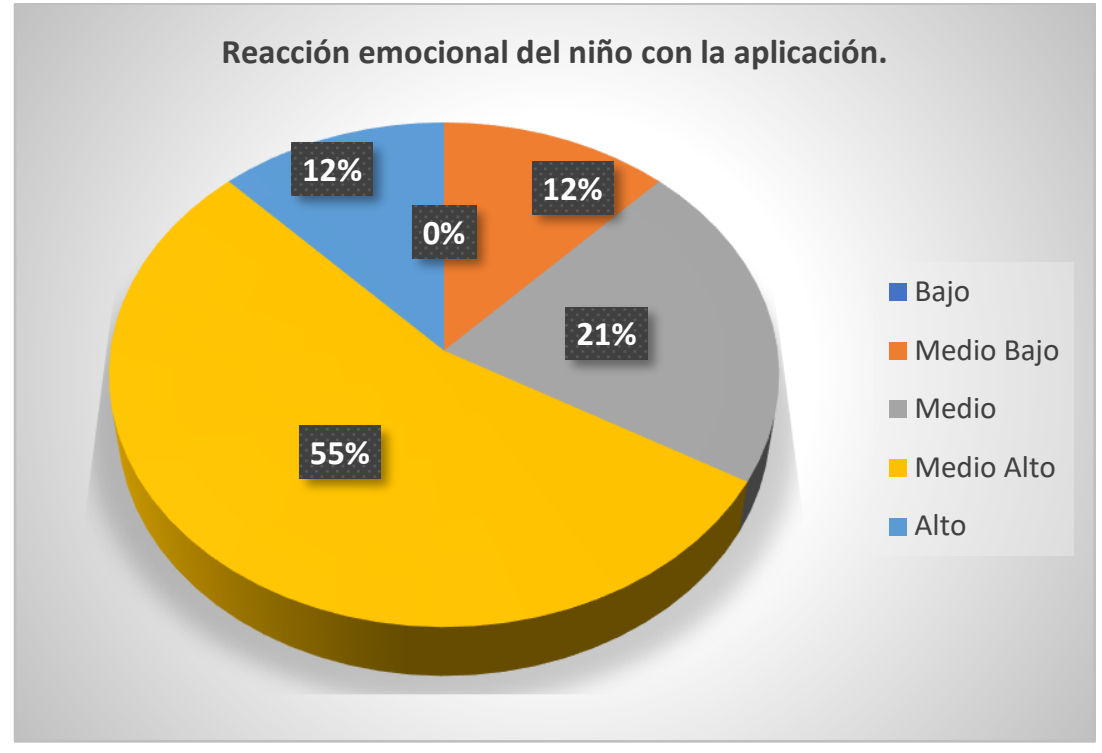

Fuente: Elaboración propia.

\section{Análisis e interpretación}

Del $100 \%$ de los padres de familia encuestados el 55\% considera que la reacción emocional del niño con la aplicación es Medio Alto, el 21\% Medio, el 12\% Alto, el 12\% Medio Bajo mientras que no se registraron frecuencias en la opción Bajo. Es decir, que en esta pregunta sigue persistiendo que la mayoría (opciones de Alto y Medio Alto) de padres de familia consideran que sus niños reacción emocionalmente de forma positiva del niño con la aplicación, considerándose también como un incentivo cognitivo para el aprendizaje.

Pregunta 4. ¿Cómo considera la interacción del niño con las actividades cotidianas asociando con la aplicación?

Tabla 6. Interacción del niño con las actividades cotidianas asociando con la aplicación

\begin{tabular}{|c|c|c|c|c|c|}
\hline \multirow[b]{2}{*}{ Preguntas } & \multicolumn{5}{|c|}{ Criterios de evaluación } \\
\hline & Bajo & $\begin{array}{c}\text { Medio } \\
\text { Bajo }\end{array}$ & Medio & $\begin{array}{c}\text { Medio } \\
\text { Alto }\end{array}$ & Alto \\
\hline $\begin{array}{l}\text { ¿Cómo considera el desarrollo del aprendizaje del } \\
\text { niño con la aplicación? }\end{array}$ & 0 & 3 & 12 & 10 & 18 \\
\hline
\end{tabular}

Fuente: Elaboración propia. 
Figura 9. Interacción del niño con las actividades cotidianas asociando con la aplicación.

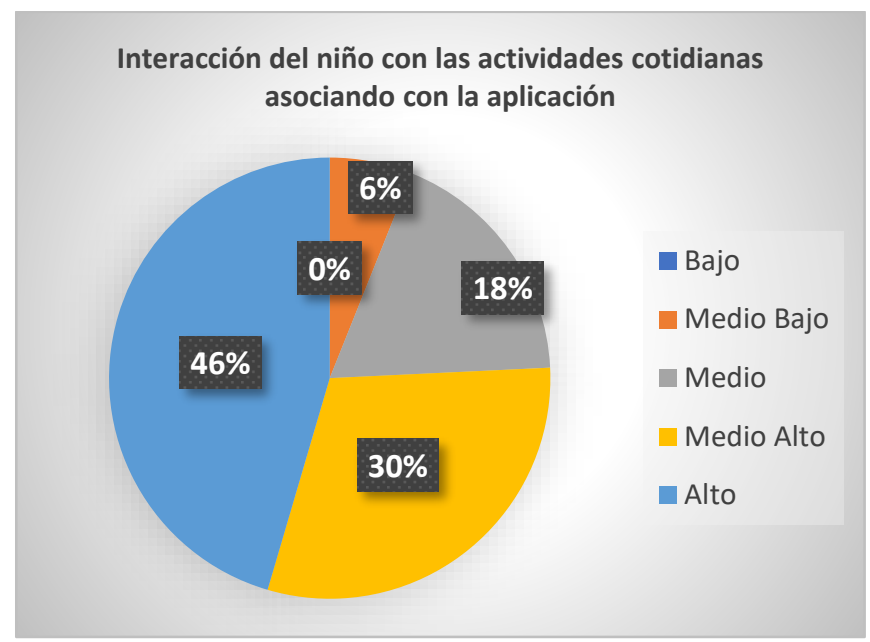

Fuente: Elaboración propia.

\section{Análisis e interpretación}

Del $100 \%$ de los padres de familia encuestados el $46 \%$ considera que la interacción del niño con las actividades cotidianas asociando con la aplicación es Alto, el 30\% Medio Alto, el 18\% Medio, el 6\% Medio Bajo mientras que no se registraron frecuencias en la opción Bajo. Por tanto, la mayoría (opciones de Alto y Medio Alto) de padres de familia consideran que sus niños reacción positivamente en las actividades diarias asociadas con lo aprendido a través de la aplicación. Es decir que existe probabilidad de un aprendizaje significativo, ya que se está poniendo en práctica en las actividades diarias.

Pregunta 5. ¿Cómo considera el desarrollo del aprendizaje del niño con la aplicación?

Tabla 7. Desarrollo del aprendizaje del niño con la aplicación

\begin{tabular}{|c|c|c|c|c|c|}
\hline \multirow[b]{2}{*}{ Preguntas } & \multicolumn{5}{|c|}{ Criterios de evaluación } \\
\hline & Bajo & $\begin{array}{c}\text { Medio } \\
\text { Bajo }\end{array}$ & Medio & $\begin{array}{l}\text { Medio } \\
\text { Alto }\end{array}$ & Alto \\
\hline $\begin{array}{l}\text { ¿Cómo considera el desarrollo del } \\
\text { aprendizaje del niño con la aplicación? }\end{array}$ & 0 & 3 & 12 & 10 & 18 \\
\hline
\end{tabular}

Fuente: Elaboración propia. 
Figura 10. Desarrollo del aprendizaje del niño con la aplicación

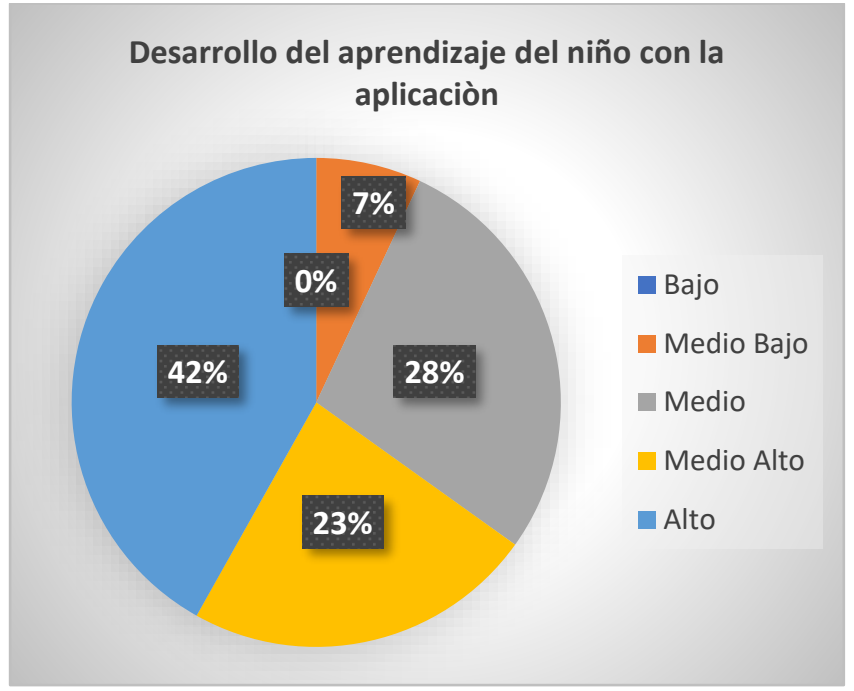

Fuente: Elaboración propia.

\section{Análisis e interpretación}

Del $100 \%$ de los padres de familia encuestados el $42 \%$ considera que el desarrollo del aprendizaje del niño con la aplicación es Alto, el 28\% Medio, el 23\% Medio Alto, el 7\% Medio Bajo; mientras que no se registraron frecuencias en la opción Bajo. Por lo que la mayoría (opciones de Alto y Medio Alto) de padres de familia consideran que sus niños están desarrollo positivamente el aprendizaje con la aplicación, pues ellos en su convivir diario fuera de las aulas, puede dar testimonio de lo afirmado.

Pregunta 6. ¿Cómo considera la facilidad de acceso a la aplicación?

Tabla 8. Facilidad de acceso a la aplicación

Criterios de evaluación

\begin{tabular}{cccccc} 
Preguntas & Bajo & $\begin{array}{c}\text { Medio } \\
\text { Bajo }\end{array}$ & Medio & $\begin{array}{c}\text { Medio } \\
\text { Alto }\end{array}$ & Alto \\
\hline $\begin{array}{c}\text { ¿Cómo considera la facilidad de acceso a la } \\
\text { aplicación? }\end{array}$ & 0 & 5 & 6 & 12 & 10 \\
\hline
\end{tabular}

Fuente: Elaboración propia. 
Figura 11. Facilidad de acceso a la aplicación

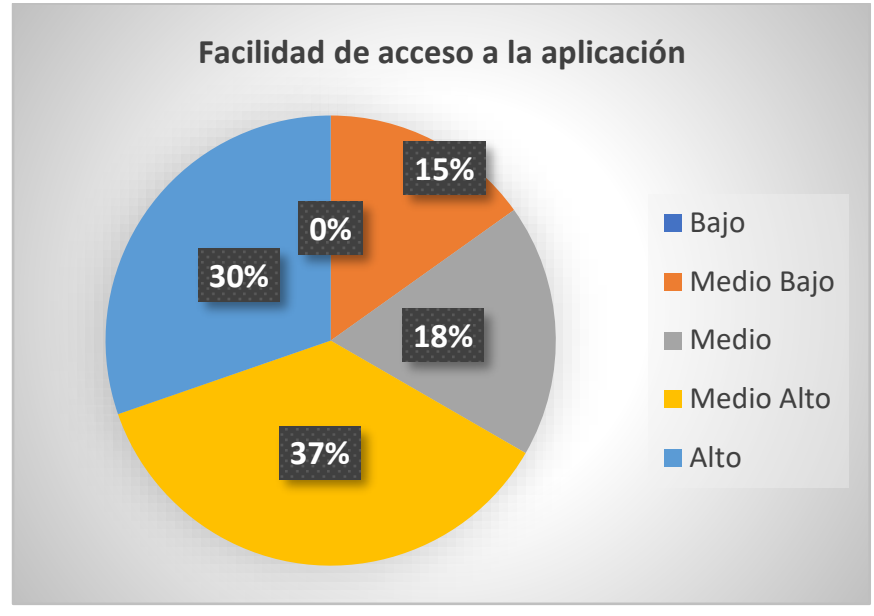

Fuente: Elaboración propia.

Del $100 \%$ de los padres de familia encuestados el $37 \%$ considera que la facilidad de acceso a la aplicación es Medio Alto, el 30\% Alto, el 18\% Medio, el 15\% Medio Bajo; mientras que no se registraron frecuencias en la opción Bajo. Por lo que la mayoría (opciones de Alto y Medio Alto) de padres de familia consideran es fácil acceder a la aplicación. Es decir que, al seguir metodologías de desarrollo de software, los niños con síndrome de Down no tuvieron problemas de acceso.

Pregunta 7. ¿Cómo considera el cambio de aprendizaje del niño con la Realidad Aumentada?

Tabla 9. Facilidad de acceso a la aplicación

\begin{tabular}{|c|c|c|c|c|c|}
\hline \multirow[b]{2}{*}{ Preguntas } & \multicolumn{5}{|c|}{ Criterios de evaluación } \\
\hline & Bajo & $\begin{array}{l}\text { Medio } \\
\text { Bajo }\end{array}$ & Medio & $\begin{array}{l}\text { Medio } \\
\text { Alto }\end{array}$ & Alto \\
\hline $\begin{array}{c}\text { ¿Cómo considera el cambio de aprendizaje del } \\
\text { niño con la Realidad Aumentada? }\end{array}$ & 0 & 9 & 1 & 9 & 14 \\
\hline
\end{tabular}

Fuente: Elaboración propia. 
Figura 12. Cambio de aprendizaje del niño con la Realidad Aumentada.

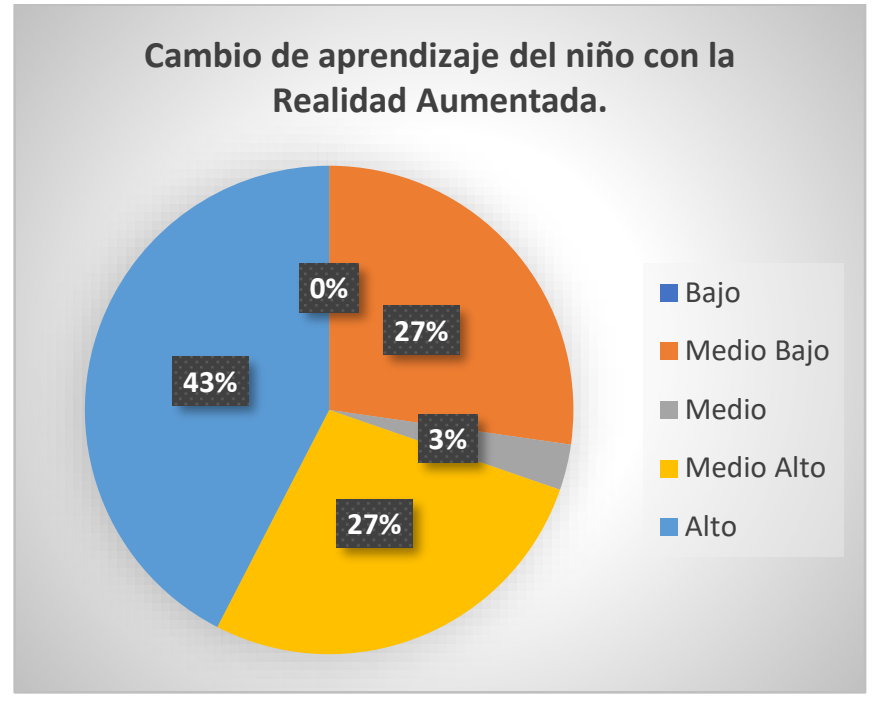

Fuente: Elaboración propia.

\section{Análisis e interpretación}

Del $100 \%$ de los padres de familia encuestados el $43 \%$ considera que el cambio de aprendizaje del niño con la Realidad Aumentada es Alto, el 27\% Medio Alto, el 27\% Medio Bajo, el 3\% Medio Bajo; mientras que no se registraron frecuencias en la opción Bajo. Por tanto, en esta pregunta también persiste lo que la mayoría (opciones de Alto y Medio Alto) de padres de familia consideran que sus niños han experimentado positivamente un cambio de aprendizaje mediante el uso de Realidad Aumentada, reafirmando que las tecnologías de la información y comunicación son herramientas valiosas para aportar en el desarrollo del aprendizaje.

Figura 13. Resultados de la evaluación.

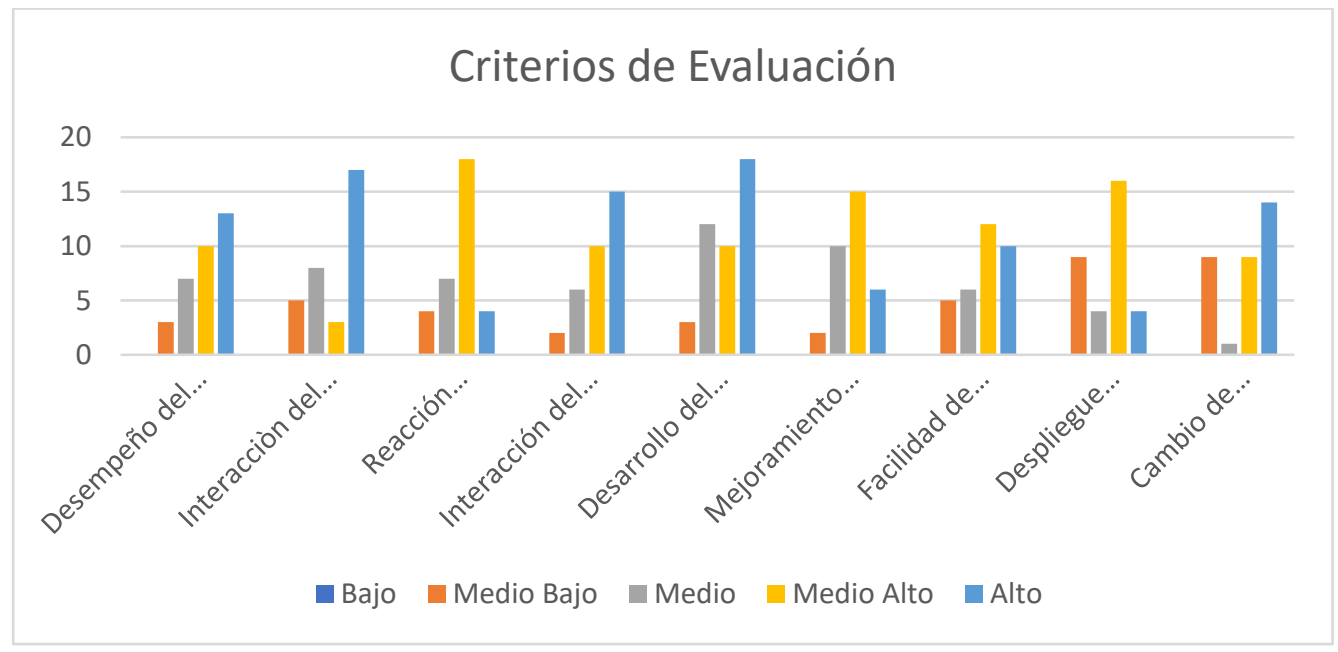

Fuente: Elaboración propia. 
Al mirar la gráfica se puede observar que las tendencias o frecuencias de respuestas son las registradas en las opciones Alto y Medio Alto, es decir que la mayoría de padres de familia consideran positivo el uso de la realidad aumentada con 3D para el aprendizaje de sus niños con síndrome de Down; mientras que las frecuencias de minoría son registradas en las opciones de Medio y Bajo Medio; opciones que deben ser atendidas mediante nuevas investigaciones con estrategias o alternativas a fin de mejorar aquellas particularidades. Lo positivo de la investigación es que no se registró en ninguna respuesta la opción bajo, por lo que todos los padres de familia de alguna forma fueron beneficiados con la investigación.

\section{Conclusiones:}

- La aplicación móvil implementada con Realidad Aumentada que incluye un personaje o avatar 3D, fue aplicada y sirvió como una herramienta de apoyo tecnológico para profesor en el proceso de formación y comunicación con los niños con Síndrome de Down, y cumplió con el objetivo de mejorar la interacción de los niños con el sistema de comunicación que utilizan diariamente.

- En el desarrollo de la aplicación móvil, se contempló la implementación de marcadores de Realidad Aumentada a través de pictogramas, que se activan cuando los niños enfoca con la cámara de su Smartphone, por su parte el pictograma mostrarán un avatar 3D animado llamativo, que emitirá un mensaje mediante movimientos que simula el mensaje que desea transmitir logrando tener un sistema de comunicación llamativo y agradable para los niños Down y al mismo tiempo incidirá en el proceso cognitivo del aprendizaje a través de la motivación y captación de información con su entorno.

- Para la evaluación de la eficiencia de la aplicación realizada se aplicó una encuesta constituida por siete preguntas, con criterios de evaluación en una escala de Likert en cada pregunta con un rango entre Bajo, Medio Bajo, Medio, Medio Alto y Alto. De los parámetros evaluados, el criterio Bajo no presentó frecuencias, es decir que fue un indicador que determinó la conformidad de los encuestados con el uso de la aplicación; mientras tanto el criterio Alto obtuvo la mayoría de respuestas favorables que indican y refuerzan la conclusión, de que los niños fueron beneficiados con el uso de la Realidad Aumentada 3D, cabe recalcar que la encuesta fue aplicada a los padres de familia responsables de la educación de los niños Down. 


\section{Referencias bibliográficas:}

Albuja, R. (2015). La enseñanza en los niños con síndrome de down. Revista familia.ec,1(1), 56.

Almazán, M. (2009). Los sistemas aumentativos y/o altenativos de la comunicación. Innovación y Experiencias. 45(6), 1-9.

Álvarez, L. (2013). Los sistemas alternativos y/o aumentativos de comunicación: la comunicación bimodal como recurso en el aula de audición y lenguaje. (Trabajo Fin de Grado).Universidad de Valladolid, España.

Basil, C. (2015).Qué son los sistemas Aumentativos y Alternativos de Comunicación?.Aragón: Aragonese portal of augmentative and alternative comunicative. Obtenido de http://arasaac.org/aac.php

Cantero, J., Morena, M., Saorín, J., Carbonell, C., \& Contero, M. (2015). Entorno de aprendizaje ubicuo con realidad aumentada y tabletas para estimular la comprensión del espacio tridimensional.Revista de Educación a Distancia.,1(37), $1-17$.

Cavanna, M. (2016). Las nuevas tecnologías facilitan el aprendizaje de niños con síndrome de down. La revista de la Fundación, compromiso y transparencia, Compromiso empresarial. Recuperado de http://www.compromisoempresarial.com/entradas/2015/04/las-nuevastecnologiasfacilitan-el-aprendizaje-de-ninos-con-sindrome-de-down/.

Góngora, M. (2011). Sistemas alternativos de comunicación. https://www.blogger.com/profile/04298767754630666615

Innovae. (2016). Qué es la Realidad Aumentada?.Innovac augmented reality. Obtenido de http://realidadaumentada.info/tecnologia/

Letelier, P. y Penadés, Ma . (2006). Métodologías ágiles para el desarrollo de software: eXtreme Programming (XP).. Técnica Administrativa.

Leyvrevh. (2012). SPC, Sistema Pictográfico de Comunicación. Gijón:Dpto. De orientación san vicente de paúl gijón. Obtenido de https://orientacionsanvicente.wordpress.com/2012/05/23/spc-sistemapictografico-decomunicacion/

Morales, B. B. Modelo de Búsqueda de Diagramas de Casos de Uso en UML a través de la Derivación de Ontologías OWL Use Cases UML Diagrams Search Model using OWL Ontologies.

NICHCY. (2010, febrero). El Síndrome de Down. Centro Nacional de Diseminación de Información para Niños con Discapacidades. https://www.isbe.net/Documents/down-syndrome-sp.pdf

Ortega-Tudela, J. M. y Gómez-Ariza, C. J. (2007). Nuevas tecnologías y aprendizaje matemático en niños con síndrome de down: generalización para la autonomía. Pixel-Bit. Revista de Medios y Educación, (29),59-72.[fecha de Consulta 8 de Enero de 2020]. ISSN: 1133-8482. Disponible en: https://www.redalyc.org/articulo.oa?id=368/36802905 
Reeduca. (2009). Características del Lenguaje. Recuperado de http://reeduca.com/estimulaciondown-lenguajec.aspx.

Ouazzani, I. (2012). Manual de creación de videjuegos con unity. (Tabajo de Titulación), Universidad Carlos iii, Madrid.

Qualcomm. (2010). Pc actual. Obtenido de http://www.pcactual.com/articulo/actualidad/noticias/mobile_world_congress/12 555/vuforia_realidad_aumentada_segun_qualcomm.html

Rodríguez, E. R. (2011). Programación educativa para escolares. Fundación Iberoamericana Down21, Abril 2011(119). 


\section{PARA CITAR EL ARTÍCULO INDEXADO.}

Centeno Aulla, H. D., Paredes Castelo, L. E., Allauca Peñafiel, M. E., Allauca Peñafiel, L. G., \& Espinoza Tinoco, L. M. (2020). Aplicación móvil en realidad aumentada para la formación y comunicación de niños con Síndrome de Down. ConcienciaDigital, 3(3), 215-236. https://doi.org/10.33262/concienciadigital.v3i3.1307

\section{DDigital}

El artículo que se publica es de exclusiva responsabilidad de los autores y no necesariamente reflejan el pensamiento de la Revista Conciencia Digital.

El artículo queda en propiedad de la revista y, por tanto, su publicación parcial y/o total en otro medio tiene que ser autorizado por el director de la Revista Conciencia Digital.

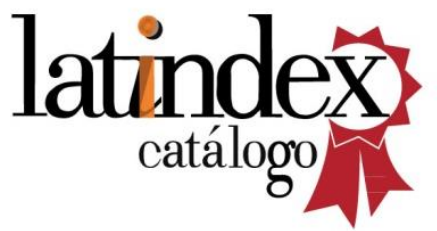

